\title{
Determination of Pollen Viability in Tomatoes
}

\author{
Aref A. Abdul-Baki \\ Vegetable Laboratory, Plant Sciences Institute, Agricultural Research Service, U.S. Department of \\ Agriculture, Beltsville, MD 20705-2350
}

Additional index words. Lycopersicon esculentum, fluorescein diacetate, fluorescence, germination, staining

\begin{abstract}
A procedure is described for determining pollen viability in tomatoes (Lycopersicon esculentum Mill.) by growing pollen in a growth medium containing $0.29 \mathrm{M}$ sucrose, $1.27 \mathrm{~mm} \mathrm{Ca}\left(\mathrm{NO}_{3}\right)_{2}, 0.16 \mathrm{~mm} \mathrm{H}_{3} \mathrm{BO}_{3}$, and $1 \mathrm{~mm} \mathrm{KNO}_{3}$ (pH 5.2) to which $0.001 \%$ fluorescein diacetate (FDA) was added. Pollen viability can be evaluated within 30 min by determining percent fluorescing pollen in a sample. The procedure further allows the determination of percent germination in vitro and pollen tube growth within 1.5 hours. Neither the germination medium nor FDA has any adverse effects on germination and pollen tube growth. Percent fluorescent pollen and percent total pollen germination were highly correlated, suggesting that fluorescence is a good measure of pollen viability. The combined fluorescencegermination procedure is simple and adapted to routine screening of many samples.
\end{abstract}

The development of reliable methods for determining the functional quality of pollen would be useful in hybridization programs (Calvert, 1964; Dempsey, 1962) and in evaluating the quality of pollen that develop under environment stresses (Shelby et al., 1978). Heslop-Harrison et al. (1984) and Picken (1984) reviewed three general approaches for evaluating pollen quality: in vivo, in vitro pollen germination and pollen tube growth, and histochemical.

The in vivo approach involves placing the pollen on the stigmas of emasculated flowers and determining the number of pollen tubes in squashed styles (Abdalla and Verkerk, 1968; Dempsey, 1970; van Koot and van Rovestijin, 1963) or the number of seeds in the mature fruit (El Ahmadi and Stevens, 1979; McGuire, 1952; Maisonneuve and Philouze, 1982). These methods are time-consuming and therefore impractical for testing many samples. Furthermore, seed set may depend not only on fertilization, but also on the post-pollination development of the ovary, pistil receptivity, and incompatibility reactions (Berry and Uddin, 1988; Heslop-Harrison et al., 1984).

In vitro germination involves germinating the pollen on artificial media and determining germinability and pollen tube growth $(\mathrm{Ab}-$ dalla and Verkerk, 1968; Charles and Harris, 1972 Hewlett, 1936; Levy et al., 1978; Weaver and Timm, 1989). The procedure requires much less time (about $1.5 \mathrm{~h}$ ) than the in vivo approach and is adapted to routine screening of many samples. However, its potential ability to predict pollen performance depends heavily on optimization of the germination medium and temperature and on the availability of an adequate amount of pollen in the tested sample (Heslop-Harrison et al., 1984).

The histochemical approach is based either on the ability of the vegetative cell of the pollen grain to stain specific constituents of that cell or on the activity of specific enzymes. Iodinepotassium iodide was applied to stain starch (Charles and Harris, 1972), aniline blue to stain starch and other polysaccharides (Dempsey, 1962; Dionne and Spicer, 1958), phyloxin-methyl green to stain cell wall (Weaver and Timm, 1989; Weaver et al., 1985), and safranin and acetocarmine to stain chromatin and ribonucleic acids (Dionne and Spicer, 1958; Rudich et al., 1977). Enzyme activity most commonly is tested by reduction of the tetrazolium group to yield the colored insoluble formazans (Cook and Stanley, 1960) and the hydrolysis of fluorescein di-

\footnotetext{
Received for publication 26 Feb. 1991. Accepted for publication 2 Dec. 1991 I thank Monika Shah for technical assistance. The cost of publishing this paper was defrayed in part by the payment of page charges. Under postal regulations, this paper therefore must be hereby marked advertisement solely to indicate this fact.
}

acetate (FDA) by esterase to yield fluorescein (Rotman and Papermaster, 1966).

The histochemical approach for determining pollen viability requires a short time (20 to 30 rein), thus making it most suitable for routine screening of many samples. However, the staining media often adversely affect the pollen and interfere with its viability. This may explain, in part, the inconsistent results of some earlier work in which data from histochemical tests and in vitro germination were compared in assessing pollen quality (Calvert, 1964). Therefore, it is essential that the germination and staining media have no adverse effects on terminability and pollen tube growth.

This work was undertaken to develop a fast and reliable method for evaluating pollen viability through the combined use of FDA and in vitro germination approaches. Pollen viability can thus be evaluated in one operation within a short time by first determining fluorescence resulting from the hydrolysis of FDA and then the terminability of the sample.

\section{Materials and Methods}

Source of pollen. Sixteen tomato plants of breeding line CL 1131-0-0-43-8-1 were grown in the greenhouse between Apr. and July 1990 under a day/night cycle of 32/26 \pm 3 C. Sixteen additional plants of 'Long Keeper' and breeding line CL 11310-0-13-0-6 were grown in a greenhouse under an optimum regime of $25 / 22 \pm 3 \mathrm{C}$ (day/night). All management operations were similar to those applied for the production of a tomato crop in greenhouses. Pollen from the first breeding line was used to establish time-courses for fluorescence development and germination and to optimize the conditions for viability measurements, using both fluorescence and in vitro germination on the same sample. Pollen from the second breeding line and 'Long Keeper' was used in viability determinations on pollen that had been stored at 6 or $25 \mathrm{C}$ for $1,2,5$, or 10 days.

Preparing the pollen sample. In preliminary tests, we established that pollen samples taken from flowers born on inflorescences one (lowest), two, and three of the same plant exhibited equal viability levels. Therefore, all subsequent determinations on pollen viability presented in this study were performed on pollen taken from flowers borne on the lowest three inflorescences. Flowers were harvested every other day, brought to the laboratory, and held at room temperature for $1 \mathrm{~h}$. Pollen from 30 flowers (70 flowers for the storage experiment) was collected in a 35-mm petri dish by touching the anthers for $5 \mathrm{sec}$ with a

Abbreviation: FDA, flourescein diacetate. 


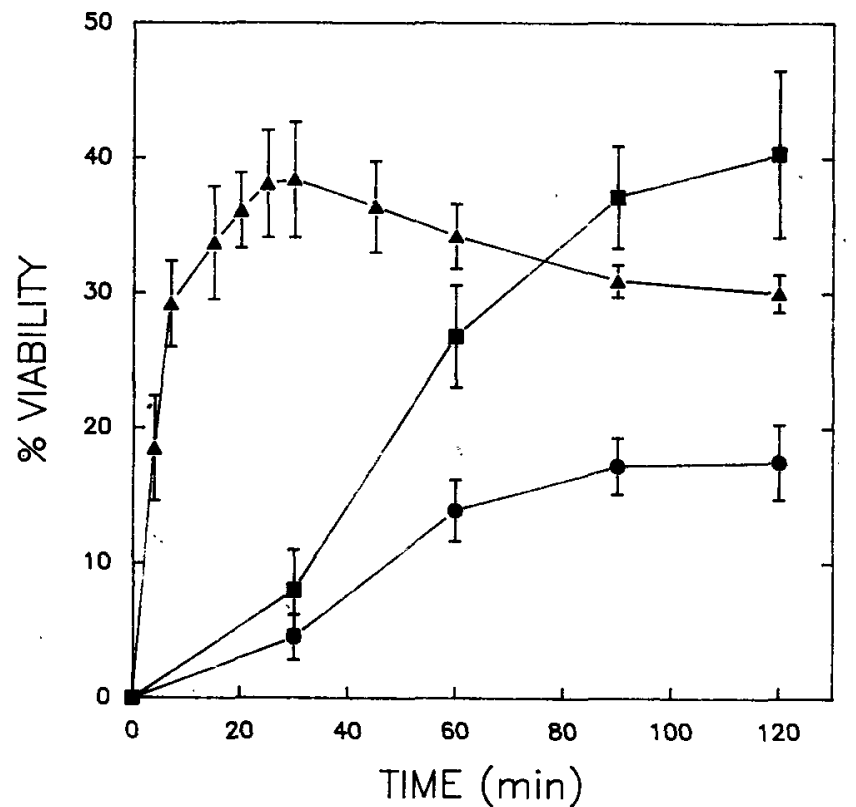

Fig. 1. Pollen viability expressed as percent normal (circles) and percent total (abnormal plus normal-appearing pollen) (squares) germination in the control sample (without FDA) and percent fluorescence (triangles) in medium with FDA. Individual points plus SE bars represent means of eight replicates.

Table 1. Fluorescence and total germination of pollen after storage at 6 or $25 \mathrm{C}$.

\begin{tabular}{lcccc}
\hline \hline & \multicolumn{2}{c}{ Storage } & & \\
\cline { 2 - 4 } Cultivar/line & $\begin{array}{c}\text { Temp } \\
\left({ }^{\circ} \mathrm{C}\right)\end{array}$ & $\begin{array}{c}\text { Duration } \\
\text { (days) }\end{array}$ & $\begin{array}{c}\text { Fluoresence } \\
(\%)\end{array}$ & $\begin{array}{c}\text { Total }^{z} \\
\text { germination }^{z} \\
(\%)\end{array}$ \\
\hline Long Keeper & 25 & 0 & $83.2^{y}$ & $85.3^{y}$ \\
& 25 & 1 & 65.7 & 63.8 \\
& 25 & 2 & 24.7 & 33.0 \\
& 25 & 5 & 22.8 & 11.5 \\
& 25 & 10 & 0 & 0 \\
& 6 & 0 & 83.2 & 85.3 \\
& 6 & 1 & 74.8 & 79.1 \\
& 6 & 2 & 64.6 & 73.6 \\
& 6 & 5 & 24.8 & 30.3 \\
& 6 & 10 & 2.7 & 4.0 \\
& 25 & 0 & 71.9 & 83.8 \\
& 25 & 1 & 55.9 & 46.2 \\
& 25 & 2 & 10.0 & 11.0 \\
& 25 & 5 & 7.1 & 6.3 \\
& 25 & 10 & 0 & 0 \\
& 6 & 0 & 71.9 & 83.8 \\
& 6 & 1 & 55.0 & 61.2 \\
& 6 & 2 & 37.6 & 29.0 \\
& 6 & 5 & 7.2 & 11.5 \\
& 6 & 10 & 2.8 & 2.7 \\
\hline
\end{tabular}

${ }^{2}$ Fluorescence at 20 to $30 \mathrm{~min}$ and germination at $90 \mathrm{~min}$ on the same sample in a growth medium containing $0.001 \%$ FDA.

${ }^{y}$ Average of two determinations.

mechanical vibrator (Dremel, Racine, Wis.). After mixing, the pollen was divided into 0.3 -mg portions, each representing a sample for one determination. Fluorescence and germination tests were performed within $4 \mathrm{~h}$ of harvesting the flowers. For determining the effect of storage temperature and duration on pollen viability, eight pollen samples ( $0.3 \mathrm{mg}$ each) of each line or cultivar were held at $25 \mathrm{C}$; another eight samples were held at 6C; and two samples were tested immediately (zero time).
Table 2. Analysis of variance on data shown in Table 1.

\begin{tabular}{lcrc}
\hline \hline Source of variation & df & F value & Significance \\
\hline Cultivar (Cult) & 1 & 140.45 & $* *$ \\
Temperature (Temp) & 1 & 87.09 & $* *$ \\
Cult $\times$ temp & 1 & 8.80 & $* *$ \\
Storage duration (days) & 4 & 720.20 & $* *$ \\
Cult $\times$ duration & 4 & 16.91 & $* *$ \\
Temp $\times$ duration & 4 & 25.20 & $* *$ \\
Cult $\times$ temp $\times$ duration & 4 & 1.91 & $* *$ \\
Type (germination vs. & 1 & 2.96 & NS \\
fluorescence) & & & \\
Cult $\times$ type & 1 & 0.09 & NS \\
Temp $\times$ type & 1 & 3.18 & NS \\
Cult $\times$ temp $\times$ type & 1 & 0.57 & NS \\
Duration $\times$ type & 4 & 2.01 & NS \\
Cult $\times$ duration $\times$ type & 4 & 2.57 & NS \\
Temp $\times$ duration $\times$ type & 4 & 1.93 & NS \\
Cult $\times$ temp $\times$ duration $\times$ type & 4 & 0.76 & NS \\
\hline NS,$*$ Nonsgnifint
\end{tabular}

NS, **Nonsignificant or significant at $P=0.05$.

Duplicate samples from each storage condition were tested after storing for 1, 2, 5, or 10 days.

Viability determinations. Viability determinations were made by two criteria on 0.3 -mg samples of pollen: 1) fluorescence in the prescence of FDA and 2) in vitro germination. The pollen samples were germinated in $35-\mathrm{mm}$ petri dishes, each containing $1.4 \mathrm{ml}$ freshly prepared Murashige and Skoog's (1962) growth medium (a liquid culture without agar) consisting of $0.29 \mathrm{M}$ sucrose, $1.27 \mathrm{~mm} \mathrm{Ca}\left(\mathrm{NO}_{3}\right)_{2}, 0.16 \mathrm{~mm} \mathrm{H}_{3} \mathrm{BO}_{3}$, and $1 \mathrm{~mm} \mathrm{KNO}_{3}$ $(\mathrm{pH}$ 5.2) for the control and the same medium containing $0.001 \%$ FDA (Sigma, St. Louis) for the fluorescence treatment. They were incubated at $27 \mathrm{C}$ for durations specified in the Results. In vitro germination was determined by scoring percent normal germination and total (includes the abnormal) germination using a Diaphot-TMD inverted microscope (Nikon, Image Systems, Columbia, Md.) and a light source from a halogen lamp. A pollen grain was considered to be germinated when the length of the pollen tube was equal to or longer than the diameter of the grain. Fluorescence in FDA was determined by scoring percent fluorescing pollen grains under an ultraviolet (UV) source provided by a mercury lamp. The principle is based on the uptake of nonfluorescing FDA by the vegetative cells of a viable pollen grain and subsequent hydrolysis by esterase to release fluorescein, which fluoresces under UV (excitation filter $=485$ $\mathrm{nm}$ and barrier filter $=520 \mathrm{rim}$ ). In contrast, nonviable cells are incapable of hydrolyzing FDA and, therefore, do not fluoresce (Heslop-Harrison and Heslop-Harrison, 1970; HeslopHarrison et al., 1984; Rotmand and Papermaster, 1966). Each determination consisted of counting 1000 to 1200 pollen grains by examining eight to 10 locations in a series of parellel traverses across the petri dish that contained the sample under test.

Analysis of variance was conducted to determine if there were differences in percent total germination and percent fluorescence. The analysis was conducted as a $2 \times 2 \times 2 \times 5$ factorial of cultivar, temperature, type (germination vs. fluorescence), and storage duration.

\section{Results and Discussion}

Pollen viability is expressed as percent normal and percent total germination (abnormal and normal-appearing grains) in the control samples as compared with that in FDA (expressed as percent fluorescence) over $120 \mathrm{~min}$ (Fig. 1). Although pollen tubes in some grains began to grow $\approx 25 \mathrm{~min}$ after being placed 

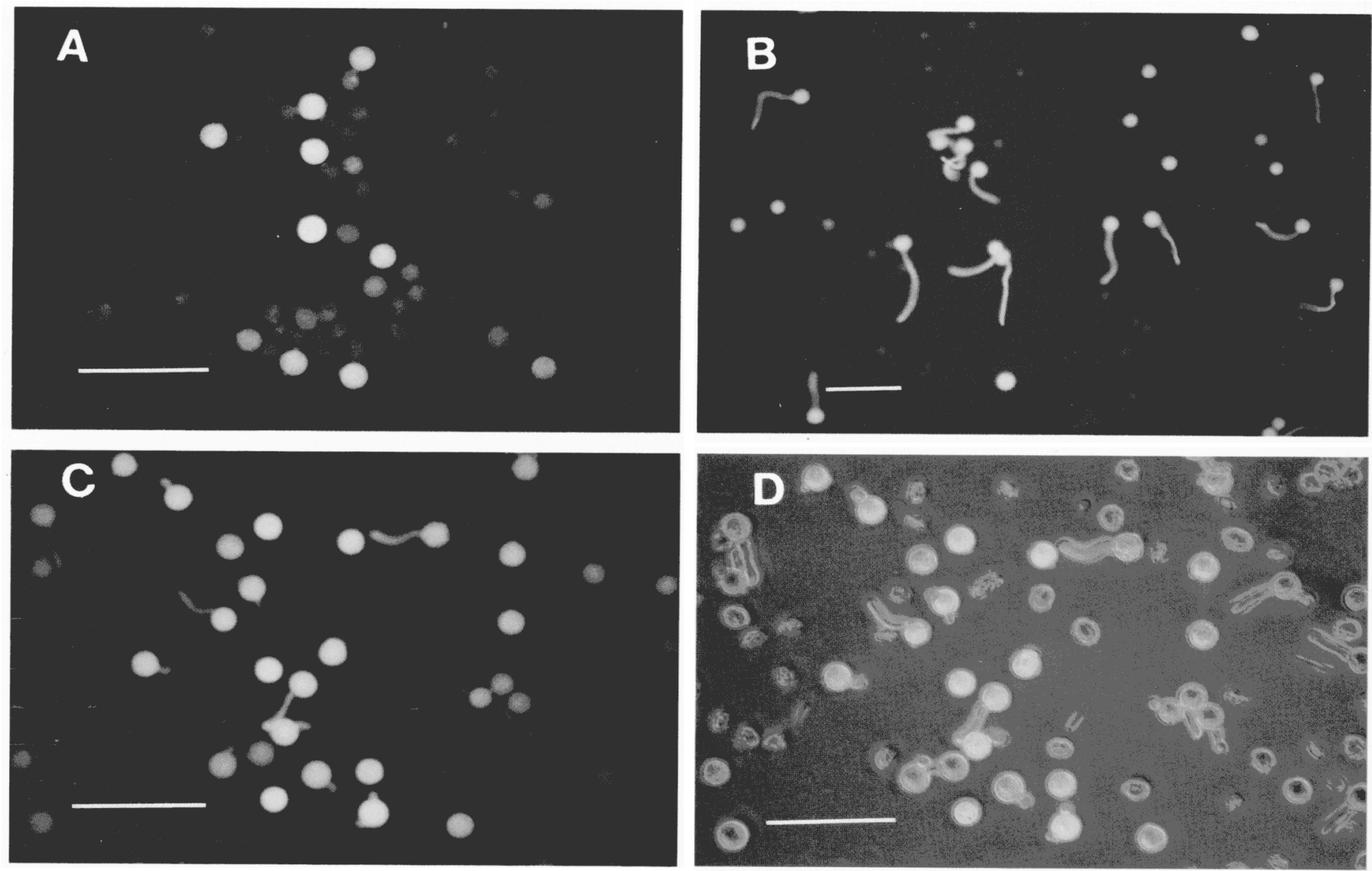

Fig. 2. Pollen viability as fluorescing pollen in a germination medium containing $0.001 \%$ fluorescein diacetate. (A) Fluorescing pollen 4 min after being placed in growth medium containing FDA. (B) Fluorescing pollen showing normal pollen tube growth after 140 min o germination and nonfluorescing, nonviable pollen. The same sample germinated for $90 \mathrm{~min}$ in the prescence of FDA with (C) subjected to UV alone and (D) lighted by both UV and a halogen lamp. Bar length $=110 \mu$.
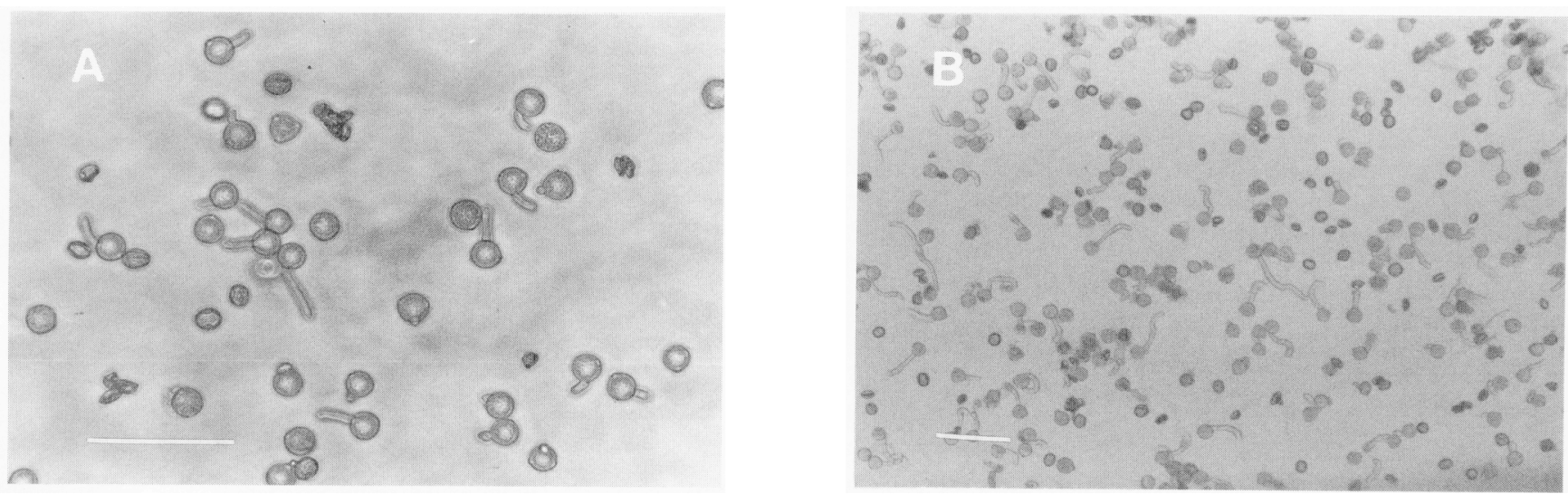

Fig. 3. Control sample (A) after 60 min germination, (B) after 100 min germination. Bar length $=110 \mu$.

in the growth medium, maximum germination of normal pollen was reached" after $90 \mathrm{~min}$. At that time, percent total germination was still increasing slightly due to the delayed germination of some abnormal pollen grains in the sample. In the FDA treatment, the pollen grains began to show some fluorescence within 2 to 4 min of being placed in the growth medium (Fig. 1). All viable pollen grains (intact and abnormal) fluoresced. However, after $30 \mathrm{~min}$ in the growth medium, fluorescence in abnormal grains began to fade away, presumably due to the leaching of fluorescein from the pollen grain into the medium and the inability of abnormal pollen to continue to hydrolyze FDA. This presumed response explains the slight decline in percent fluorescence after $30 \mathrm{~min}$ (Fig. 1). The data suggest that maximum fluorescence in FDA at 30 min represents percent total viability 
and appears comparable to percent total germination at $90 \mathrm{~min}$ in the control sample.

No significant difference was found between percent in vitro pollen germination at $90 \mathrm{~min}$ and percent pollen fluorescence at 20 to $30 \mathrm{~min}$ (Tables 1 and 2). Likewise, there were no significant interactions between viability tests and other factors (temperature and storage duration). Pollen viability decreased significantly over storage time, and pollen stored better at 6 than at $25 \mathrm{C}$. The correlation between the FDA method and the in vitro germination method in assessing viability of tomato pollen was strong $(r=0.97)$ and highly significant $(\mathrm{P}<0.001)$. The results are in agreement with the findings of Heslop-Harrison and Heslop-Harrison (1970) and Heslop-Harrison et al. (1984). However, their microfluorimetry method focused on membrane integrity and involved the measurement of fluorescence in individual pollen grains and the leaching of fluorescein into the incubation medium. Their procedure requires considerable time to determine fluorescence in several hundred grains per sample. Furthermore, prolonged exposure to $\boldsymbol{W}$ light during scoring reduces the intensity of fluorescence and viability. In addition, Heslop-Harrison et al. (1984) used a medium that was high in sucrose, lacking in boron, and the FDA concentration was 40 times higher than that used in the present study.

The use of FDA to assess pollen viability by determining percent fluorescing pollen in a sample within 30 min of incubating in a growth medium offers a simple and reliable method that is adapted to routine screening of many samples. Neither the growth medium nor FDA, at the concentration used, had any adverse effects on germination and pollen tube growth (compare Figs. 2C and $3 \mathrm{~A}$ and Figs. 2B and 3B). . Several stains (phenosafranin, lacmoid, trypan blue, and 2,3,5-triphenyl-tetrazolium chloride) were also evaluated in preliminary experiments and proven to damage pollen membranes severely and inhibit germination at the concentrations recommended for staining.

Two drawbacks arise from the use of FDA in pollen viability determinations. The first is difficulty in recognizing some of the nonfluorescing grains that appear almost as dark as the background (Fig. 2 B and C). Underscoring nonfluorescing grains tends to overestimate the fluorescing grains in the sample. The second is the inability to distinguish between fluorescing pollen grains that will germinate normally and those that will give rise to abnormal germination, because both fluoresce. The first problem can easily be overcome by subjecting the sample during scoring to both $\boldsymbol{W}$ and low-intensity light from a halogen lamp (Fig. $2 \mathrm{C}$ and D). Under dual illumination, fluorescing grains can still be recognized by their yellow fluorescence, and nonfluorescing grains become clearly visible (compare Fig. 2C with 2D). However, resolving the fluorescing pollen in a sample into those that will germinate normally and those that will give rise to abnormal germination requires extending the germination time to $90 \mathrm{~min}$ and examining the sample under a light source from the halogen lamp. Under these conditions, pollen grains with damaged membranes as well as abnormal pollen (such as those with two pollen tubes) can easily be distinguished from intact pollen that exhibit normal germination. Damaged and abnormal pollen eventually release their content into the growth medium, shrink, and lose their fluorescence.

Because of the increase in pollen abnormalities under stressful conditions, it is recommended that the fluorescence measurement be made at $30 \mathrm{~min}$ followed by a second measurement at 90 min under dual light to gain specific information about the integrity of the germinating pollen and the frequency of abnormalities in the tested sample.
The use of FDA in the Murashige-Skoog growth medium for evaluating pollen viability provides a fast and reliable technique in which viability can be determined both by fluorescence within $30 \mathrm{~min}$ and by the actual in vitro germination after $90 \mathrm{~min}$.

\section{Literature Cited}

Abdalla, A.A. and K. Verkerk. 1968. Growth, flowering and fruit-set of the tomato at high temperature. Neth. J. Agr. Sci. 16:71-76.

Barry, S.Z. and M.R. Uddin. 1988. Set in tomato cultivars and selected germplasm. HortScience 23:606-608.

Calvert, A. 1964. Pollen viability, germination and tube growth in tomato (Lycopersicon esculentum Mill.). A review of the literature. Ann. Rpt. Glasshouse Crops Res. Inst. for 1963. p. 131-142.

Charles, W.B. and R.E. Harris. 1972. Tomato fruit-set at high and low temperatures. Can. J. Plant Sci. 52:497-506.

Cook, S.A. and R.G. Stanley. 1960. Tetrazolium chloride as indicator of pine pollen gerrninability. Silvae. Genet. 9:134-136.

Dempsey, W.H. 1962. Pollen tube growth in vivo as a measure of pollen viability. Science 138:436-437.

Dempsey, W.H. 1970. Effects of temperature on pollen germination and tube growth. Tomato Genet. Coop. Rpt. 20:15-16.

Dionne, L.A. and P.B. Spicer. 1958. Staining germinating pollen and pollen tubes. Stain Technol. 33:15-17.

El Ahmadi, A.B. and M.A. Stevens. 1979. Reproductive responses of heat-tolerant tomatoes to high temperatures. J. Amer. Soc. Hort. Sci. 104:686-691.

Heslop-Harrison, J. and Y. Heslop-Harrison. 1970. Evaluation of pollen viability by enzymatically induced fluorescence: Intracellular hydrolysis of fluorescein diacetate. Stain Technol. 45:115-120.

Heslop-Harrison, J., Y. Heslop-Harrison, and K.R. Shivanna. 1984. The evaluation of pollen quality, and a further appraisal of the fluorochromatic (FCR) test procedure. Theor. Appl. Genet. 67:367-375.

Hewlett, F.S. 1936. The effect of carbohydrate and nitrogen deficiency upon microsporogenesis and the development of the male gametophyte in the tomato, Lycopersicon esculentum Mill. Ann. Bet. (London) 50:767-803.

Levy, A., H.D. Rabinowitch, and N. Keder. 1978. Morphological and physiological characters affecting flower drop and fruit set of tomatoes at high temperatures. Euphytica 27:211-218.

Maisonneuve, B. and J. Philouze. 1982. Action des basses temperatures nocturnes sur une collection varietale de tomate (Lycopersicon esculentum Mill.). I. Etude de la production de fruits et de la valeur fecondante du pollen. Agronomic 2:443-452.

McGuire, D.C. 1952. Storage of tomato pollen. Proc. Amer. Soc. Hort. Sci. 60:419-424.

Murashige, T. and F. Skoog. 1962. A revised medium for rapid growth and bioassays with tobacco tissue cultures. Physiol. Plant. 15:474-497.

Picken, A.J.F. 1984. A review of pollination and fruit-set in the tomato (Lycopersicon esculentum Mill.). HortScience 59(1):1-13.

Rotman, B. and B.W. Papermaster. 1966. Membrane properties of living mammalian cells as studied by enzymatic hydrolysis of fluorogenic esters. Proc. Natl. Acad. Sci. USA. 55:134-141.

Rudich, J., E. Zamski, and Y. Regev. 1977. Genotypic variation for sensitivity to high temperature in the tomato: Pollination and fruitset. Bet. Gaz. 138:440-452.

Shelby, R.A., W.H. Greenleaf, and C.M. Peterson. 1978. Comparative floral fertility in heat tolerant and heat sensitive tomatoes. J. Amer. Soc. Hort. Sci. 103:778-780.

Sugiyama, T., S. Iwahori, and K. Takahashi. 1966. Effect of high temperature on fruit setting of tomato under cover. Acts Hort. 4:63-69.

van Hoot, I. and W. van Ravestijin. 1963. The germination of tomato on the stigma. Proc. 16th Intl. Hort. Congr., 1962.2:452-461.

Weaver, M.L. and H. Timm. 1989. Screening tomato for high temperature tolerance through pollen viability tests. HortScience 24(3):493-495.

Weaver, M.L., H. Timm, M.J. Silbernagel, and D.W. Burke. 1985. Pollen staining and high temperature tolerance of bean. J. Amer. Soc. Hort. Sci. 110(6):797-799. 